\title{
Hot subdwarfs from the ESO supernova la progenitor survey ${ }^{\star}$ II. Atmospheric parameters of subdwarf $O$ stars
}

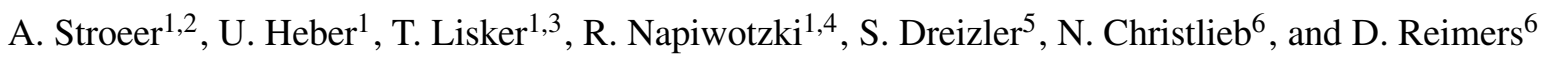 \\ ${ }^{1}$ Dr. Remeis-Sternwarte Bamberg, Astronomical Institute of the University of Erlangen-Nürnberg, Sternwartstraße 7, \\ 96049 Bamberg, Germany \\ e-mail: heber@sternwarte.uni-erlangen.de \\ 2 School of Physics and Astronomy, University of Birmingham, Edgbaston, Birmingham B29 4PT, UK \\ 3 Astronomical Institute, Dept. of Physics and Astronomy, University of Basel, Venusstrasse 7, 4102 Binningen, Switzerland \\ ${ }^{4}$ Centre for Astrophysics Research, University of Hertfordshire, College Lane, Hatfield AL10 9AB, UK \\ 5 Institut für Astrophysik, University of Göttingen, Friedrich-Hund-Platz 1, 37077 Göttingen, Germany \\ ${ }^{6}$ Hamburger Sternwarte, Universität Hamburg, Gojensbergweg 112, 21029 Hamburg, Germany
}

Received 8 May 2006 / Accepted 20 September 2006

\section{ABSTRACT}

\begin{abstract}
Aims. We address the origin and evolutionary status of hot subdwarf stars by studying the optical spectral properties of 58 subdwarf $\mathrm{O}(\mathrm{sdO})$ stars. Combining them with the results of our previously studied subdwarf B (sdB) stars, we aim at investigating possible evolutionary links.

Methods. We analyse high-resolution $(R>18000)$, high-quality optical spectra of sdO stars obtained with the ESO VLT UVES echelle spectrograph in the course of the ESO Supernova Ia Progenitor Survey (SPY). Effective temperatures, surface gravities, and photospheric helium abundances are determined simultaneously by fitting the profiles of hydrogen and helium lines using dedicated synthetic spectra calculated from an extensive grid of NLTE model atmospheres.

Results. We find spectroscopic or photometric evidence for cool companions to eight sdO stars, as well as a binary consisting of two sdO stars. A clear correlation between helium abundances and the presence of carbon and/or nitrogen lines is found: below solar helium abundance, no sdO star shows $\mathrm{C}$ or $\mathrm{N}$ lines. In contrast, $\mathrm{C}$ and/or $\mathrm{N}$ lines are present in the spectra of all sdO stars with supersolar helium abundance. We thus use the solar helium abundance to divide our sample into helium-deficient and helium-enriched sdO stars. While helium-deficient $\mathrm{sdO}$ stars are scattered in a wide range of the $T_{\mathrm{eff}}-\log (\mathrm{g})$-diagram, most of the helium-enriched sdO stars cluster in a narrow region at temperatures between 40000 and $50000 \mathrm{~K}$ and gravities between $\log g=5.5$ and 6.0 .

Conclusions. An evolutionary link between $\mathrm{sdB}$ stars and $\mathrm{sdO}$ stars appears plausible only for the helium-deficient sdO stars. They probably have evolved away from the extreme horizontal branch; i.e., they are the likely successors to sdB stars. In contrast, the atmospheric properties of helium-enriched $\mathrm{sdO}$ stars cannot be explained with canonical single-star evolutionary models. Alternative scenarios for both single-star (late hot flasher) and binary evolution (white-dwarf merger; post-RGB evolution) fail to reproduce the observed properties of helium-enriched sdO stars in detail. While we regard the post-RGB scenario as inappropriate, the white-dwarf merger and the late hot-flasher scenarios remain viable to explain the origin of helium-enriched sdO stars.
\end{abstract}

Key words. binaries: spectroscopic - stars: abundances - stars: atmospheres - stars: fundamental parameters stars: horizontal-branch - stars: subdwarfs

\section{Introduction}

Hot subluminous stars are an important population of faint blue stars at high galactic latitudes closely related to the horizontal branch. A proper spectral classification of hot subluminous stars is rendered difficult by the diversity of the helium line spectra. They can be grouped roughly into the cooler sdB stars, whose spectra typically display no or only weak helium lines, and the hotter sdO stars, which have a higher helium abundance on average and can even be dominated by helium. The former have recently been studied extensively because they are common enough to account for the UV excess observed in early-type galaxies. Pulsating sdB stars are important tools for asteroseismology (Charpinet et al. 2004), and sdB stars in close binaries

* Based on observations collected at the Paranal Observatory of the European Southern Observatory for programme No. 165.H-0588(A) and 167.D-0407(A). may qualify as Supernova Ia progenitors (Maxted et al. 2000; Geier et al. 2006).

Subluminous B stars have been identified as extreme horizontal branch (EHB) stars (Heber 1986); i.e., they are core helium-burning stars with hydrogen envelopes that are too thin to sustain hydrogen burning (unlike normal HB stars). Therefore they evolve directly to the white-dwarf cooling sequence by avoiding the asymptotic giant branch (AGB). While the sdB stars spectroscopically form a homogeneous class, a large variety of spectra is observed among sdO stars (Heber \& Jeffery 1992; Heber et al. 2006). Most subluminous B stars are helium poor, whereas only a relatively small fraction of sdO stars are.

Ever since the pioneering work by Greenstein \& Sargent (1974), the helium-rich sdO stars were believed to be linked to the evolution of the hydrogen-rich subluminous B stars. Any evolutionary link between subluminous B and O stars, however, is difficult to explain since the physical processes driving a transformation of a hydrogen-rich star into a helium-rich one remain 
obscure. The convective transformation has been explored by Wesemael et al. (1982), as well as by Groth et al. (1985). While the former found helium convection even at subsolar helium abundances, which mixes helium from deeper layers into the photosphere, the latter concluded that a helium-driven convection zone develops only in helium-rich atmospheres. If the latter is true, convective transformation would not work. Non-standard evolutionary models were introduced to explain the formation of sdO stars (e.g. Sweigart 1997b; Brown et al. 2001; Moehler et al. 2004). In particular, the late hot flasher scenario predicts that the core helium flash may occur when the star has already left the red giant branch (RGB) and is approaching the white-dwarf cooling sequence (delayed He core flash). During the flash, He and $\mathrm{C}$ are dredged-up to the surface. Hydrogen is mixed into deeper layers and burnt. The remnant is found to lie close to the helium main sequence, i.e. at the very end of the theoretical extreme horizontal branch.

The fraction of sdB stars in short period binaries (periods less than ten days) is high. Maxted et al. (2001) found 2/3 of their sdB sample (drawn from the Palomar Green survey) were such binaries, whereas somewhat lower fractions of $40 \%$ and $48 \%$ were found recently for the SPY sample (Napiwotzki et al. 2004) and for a sample drawn from the Edinburgh-Cape cata$\log$ (Morales-Rueda et al. 2006), respectively. Obviously, binary evolution plays an important role in the formation of sdB stars and possibly also in that of the sdO stars. A recent population synthesis study (Han et al. 2003) identified three channels for forming sdB stars: (i) one or two phases of common envelope evolution, (ii) stable Roche-lobe overflow, and (iii) the merger of two helium-core white dwarfs. The latter could explain the population of single stars. The simulations by Han et al. (2003) cover the observed parameter range of sdB stars (see Lisker et al. 2005, henceforth Paper I).

The ESO Supernova Ia Progenitor SurveY (SPY, Napiwotzki et al. 2001) has obtained VLT/UVES spectra for over 1000 white-dwarf candidates to test possible scenarios for type Ia supernovae by searching for double degenerate white-dwarf binary systems close to the Chandrasekhar mass limit. Many of the target stars of SPY came from the Hamburg ESO survey (Wisotzki et al. 1996). SPY also observed 137 hot subluminous stars that entered the target sample because they were previously classified as white dwarfs. Seventy-six of these stars are now classified as sdB/sdOB, and 58 as O-type subdwarfs (Christlieb et al. 2001; Lisker 2003).

The data for our hot subdwarf sample are of unprecedented quality and homogeneity. Spectral analyses of all sdB stars from that sample have already been presented in Paper I in order to test evolutionary models, in particular the binary population models of Han et al. (2003). Two diagnostic tools - the effective temperature $\left(T_{\text {eff }}\right)$ vs. surface gravity $(\log (g))$ diagram and the cumulative luminosity function - yielded conflicting results. Moreover, the models of Han et al. (2003) predicted that some EHB stars are hotter than the sdB stars contained in the sample of Paper I. This led us in Paper I to the conclusion that their sample of hot subdwarfs may not be sufficiently complete to describe the whole parameter range covered by the simulations and that it needs to be complemented by a similar analysis of subdwarf O stars.

In this paper we focus on the subdwarf $\mathrm{O}$ spectra from the SPY sample and present the spectral analysis of the highresolution spectra using state-of-the-art NLTE model atmospheres. This enables us to address the still open question about the evolutionary status of hot subdwarfs. We combine our results for the SPY sdO stars with those for the SPY sdB stars from Paper I to cover the entire stellar atmospheric parameter range with the same high accuracy. This allows a detailed comparison with evolutionary model predictions for the first time, particularly in the regime of hotter temperatures and higher helium abundances than in previous studies of sdB stars alone.

The paper is organised as follows. In Sect. 2 we outline the available data and explain our criteria for spectral classification. Section 3 briefly describes the construction of a new NLTE model atmosphere grid. In Sect. 4 we explain the derivation of stellar parameters in detail by fitting model atmospheres to observed spectral lines and present the results in Sect. 5. Various evolutionary scenarios are tested in Sect. 6, leading to a discussion and summary in Sect. 7.

\section{Observation, data reduction, and spectral classification}

Observations were obtained at the ESO Very Large Telescope with UT2 (Kueyen) equipped with the UV-Visual Echelle Spectrograph (UVES). A slit width of 2'. 1 was used, resulting in a resolving power of 18500 (spectral resolution of $0.36 \AA$ at $\mathrm{H} \alpha$ ) or better. Wavelength coverage of $3300-6650 \AA$ is achieved, with gaps at 4500-4600 ̊ and 5600-5700 ̊ (Napiwotzki et al. 2001; Koester et al. 2001). For most of the stars, two exposures were taken on different nights, since SPY was originally intended to search for radial velocity (RV) variable objects. The spectra were then reduced with a procedure developed by C. Karl using the ESO MIDAS software package, partly based on the UVES pipeline developed at ESO.

Section 2.1 sees the definition of our spectral classification as used in this paper. Section 2.2 explains the search modes for companion stars.

\subsection{Spectral classification}

For many years attempts have been made to establish a consistent classification of hot subdwarfs (Green et al. 1986; Jeffery et al. 1997; Drilling et al. 2003) that applies to lower resolution spectra. A transfer of these schemes to the spectra of higher resolution would be desirable. However, in the case of our UVES spectra, we lack the He II $4542 \AA$ line due to a wavelength gap between the blue and the red arms of the spectrograph. Since this line plays a crucial role in the classification scheme of Drilling et al. (2003), we cannot apply this scheme to our data. Instead we aim for a less detailed classification strategy.

In the process of classifying all SPY spectra (Lisker 2003), we therefore just separated the spectra into hot white dwarfs (of various subtypes), sdB, He-sdB, sdO, and He-sdO stars by visual inspection and comparison with spectra of prototypical stars. The terms $\mathrm{He}-\mathrm{sdB} / \mathrm{He}-\mathrm{sdO}$ were introduced to mark extremely helium-rich sdB/sdO stars (Ahmad \& Jeffery 2003, 2005). Note that we did not use the intermediate class of sdOB stars introduced by Baschek \& Norris (1975), but instead subsumed them in the term sdB. For our programme stars in the present paper, we continue to use only the general term sdO, in order to prevent any bias from the qualitative visual classification entering our quantitative study of atmospheric parameters.

It is worthwhile noting that our classification and the one of Green et al. (1986) do not agree, as becomes apparent from several stars that we have in common (see Table 1 of this paper and Table 1 of Paper I). Seven programme stars (four sdB and three sdO) have been misclassified as white dwarfs by Green et al. (1986). While none of the sdB stars in Paper I has been 
Table 1. Results of the quantitative spectral analysis of the ESO SPY sdO stars sorted by increasing helium abundance (log ( $y)$ ). "Outliers", i.e. values with relative errors between two or more exposures above the 2- $\sigma$ level of the global relative error, are flagged by an asterisk.

\begin{tabular}{|c|c|c|c|c|c|c|c|c|c|}
\hline Object & ICRS 2000.0 & $\begin{array}{l}B \\
\text { mag }\end{array}$ & $\begin{array}{l}M_{V} \\
\text { mag }\end{array}$ & $\begin{array}{l}T_{\text {eff }} \\
{[\mathrm{K}]}\end{array}$ & $\begin{array}{l}\log (g) \\
{\left[\mathrm{cm} \mathrm{s}^{-2}\right]}\end{array}$ & $\log (y)$ & $\begin{array}{l}\log (L) \\
{\left[L_{\odot}\right]}\end{array}$ & $\begin{array}{l}\mathrm{CN} \\
\text { class }\end{array}$ & Rem. \\
\hline \multicolumn{10}{|l|}{ helium deficient: } \\
\hline HE 1059-2735 & $11: 01: 24.95-27: 51: 42.9$ & $15.21^{a}$ & 3.11 & 40966 & 5.38 & -3.62 & 2.16 & 0 & 2 \\
\hline HE 1130-0620 & $11: 32: 41.60-06: 36: 54.4$ & $15.76^{a}$ & 4.05 & 48122 & 5.84 & -3.07 & 1.98 & 0 & 1 \\
\hline HE 1237-1408 & $12: 39: 56.64-14: 24: 48.4$ & $15.97^{a}$ & 3.02 & 39683 & 5.33 & -2.99 & 2.15 & 0 & $1,2,5,8$ \\
\hline HE $1318-2111$ & $13: 21: 15.66-21: 27: 18.5$ & $14.48^{a}$ & 3.31 & $36254^{*}$ & 5.42 & -2.91 & 1.92 & 0 & 2,5 \\
\hline HE $1115-0631$ & $11: 18: 11.69-06: 47: 33.2$ & $14.77^{a}$ & 4.17 & 40443 & 5.80 & -2.59 & 1.72 & 0 & 1 \\
\hline PG 0026+136 & $00: 28: 52.33+13: 54: 45.8$ & $15.74^{1}$ & 3.17 & 38830 & 5.38 & -2.39 & 2.07 & 0 & 1 \\
\hline EC 09445-0905 & 09:47:03.39-09:19:50.5 & $15.69^{a}$ & 4.59 & 73862 & 6.22 & -2.08 & 2.28 & 0 & 2,4 \\
\hline HE 1423-0119 & $14: 25: 51.29-01: 33: 17.4$ & $16.56^{a}$ & 3.12 & 52662 & 5.50 & -1.61 & 2.48 & 0 & 1 \\
\hline HE $1513-0432$ & $15: 16: 19.17-04: 43: 58.0$ & $16.04^{a}$ & 4.41 & 42699 & 5.92 & $-1.36^{*}$ & 1.69 & 0 & 1,7 \\
\hline HE 0040-4838 & $00: 42: 31.08-48: 22: 16.2$ & 16.07 & 3.79 & 41823 & 5.67 & -1.35 & 1.91 & 0 & \\
\hline HE 1047-0637 & $10: 50: 28.79-06: 53: 25.9$ & $14.42^{a}$ & 1.49 & 60650 & 5.03 & -1.34 & 3.39 & 0 & 1,5 \\
\hline HE $1356-1613$ & $13: 59: 12.52-16: 28: 01.8$ & 16.18 & 4.11 & $55925^{*}$ & 5.93 & -1.30 & 2.15 & 0 & 5 \\
\hline HE 1106-0942 & 11:09:08.22-09:58:48.6 & 16.34 & 4.92 & $79742^{*}$ & 6.40 & -1.03 & 2.27 & 0 & 1 \\
\hline \multicolumn{10}{|l|}{ helium enriched: } \\
\hline HE 1238-1745 & $12: 41: 01.16-18: 01: 59.0$ & $14.31^{a}$ & 3.83 & 38219 & 5.64 & -0.55 & 1.78 & $\mathrm{~N}$ & 2,5 \\
\hline HE $1258+0113$ & $13: 00: 59.23+00: 57: 11.7$ & $16.23^{b}$ & 3.80 & 39359 & 5.64 & -0.53 & 1.83 & $\mathrm{~N}$ & $1,5,7$ \\
\hline HE 2218-2026 & $22: 21: 13.02-20: 11: 17.6$ & 16.28 & 4.40 & 38330 & 5.87 & -0.35 & 1.56 & $\mathrm{C} \& \mathrm{~N}$ & \\
\hline HE $1135-1134$ & $11: 38: 10.66-11: 51: 03.8$ & 15.45 & 3.88 & 40079 & 5.68 & -0.26 & 1.82 & $\mathrm{~N}$ & 1 \\
\hline HE $1136-2504$ & $11: 39: 10.21-25: 20: 55.5$ & $13.83^{a}$ & 4.25 & 41381 & 5.84 & -0.16 & 1.72 & $\mathrm{~N}$ & 2,5 \\
\hline HE $1310-2733$ & $13: 12: 50.65-27: 49: 03.1$ & 14.38 & 3.76 & 40000 & 5.63 & -0.15 & 1.87 & $\mathrm{~N}$ & 2,5 \\
\hline HE 2359-2844 & 00:01:38.48 -28:27:42.8 & 15.7 & 3.85 & 38325 & 5.65 & -0.15 & 1.77 & $\mathrm{C} \& \mathrm{~N}$ & 3 \\
\hline PG 2204+070 & $22: 07: 16.20+07: 18: 36.0$ & $15.74^{4}$ & 3.72 & 40606 & 5.62 & 0.07 & 1.90 & $\mathrm{~N}$ & $1,4,8$ \\
\hline HE $1256-2738$ & $12: 59: 01.48-27: 54: 19.3$ & $16.29^{a}$ & 4.04 & 40029 & 5.68 & 0.09 & 1.82 & $\mathrm{C} \& \mathrm{~N}$ & 2,5 \\
\hline HE $2203-2210$ & $22: 06: 29.38-21: 56: 00.0$ & 15.04 & 4.95 & 47343 & 6.20 & 0.45 & 1.59 & $\mathrm{C} \& \mathrm{~N}$ & \\
\hline HE 1142-2311 & $11: 44: 50.15-23: 28: 18.0$ & $15.37^{a}$ & 3.86 & 54718 & 5.80 & 0.68 & 2.21 & $\mathrm{C}^{d}$ & $2,5,6$ \\
\hline HE 0111-1526 & $01: 13: 38.24-15: 11: 02.6$ & $14.59^{a}$ & 3.86 & 39152 & 6.31 & 0.83 & 1.16 & $\mathrm{C} \& \mathrm{~N}$ & 3,8 \\
\hline HE $1251+0159$ & $12: 54: 08.35+01: 43: 24.0$ & $15.24^{a}$ & 4.47 & 48208 & 5.98 & 1.03 & 1.80 & $\mathrm{C}$ & 1 \\
\hline HE $1511-1103$ & 15:14:17.04-11:14:13.6 & $14.78^{a}$ & 3.88 & 42298 & 5.68 & 1.10 & 1.91 & $\mathrm{C} \& \mathrm{~N}$ & 1,5 \\
\hline HE $1430-0815$ & $14: 33: 36.93-08: 28: 24.8$ & $15.69^{a}$ & 2.32 & 61011 & 5.26 & 1.17 & 2.97 & $\mathrm{C}$ & $1,2,7,8$ \\
\hline HE 1203-1048 & $12: 05: 56.59-11: 05: 29.4$ & 15.69 & 3.98 & 45439 & $5.91^{*}$ & $1.36^{*}$ & 1.91 & $\mathrm{C}$ & 1 \\
\hline HE $1446-1058$ & $14: 49: 24.49-11: 11: 19.0$ & $15.00^{a}$ & 3.91 & 45000 & 5.76 & 1.37 & 1.94 & $\mathrm{C} \& \mathrm{~N}$ & 2 \\
\hline HE 0342-1702 & $03: 44: 58.82-16: 52: 42.2$ & 14.75 & 4.18 & 41914 & 5.78 & 1.40 & 1.79 & $\mathrm{~N}$ & \\
\hline HE $0952+0227$ & $09: 55: 34.57+02: 12: 47.9$ & 14.72 & 3.84 & $46524^{*}$ & 5.75 & 1.41 & 2.00 & $\mathrm{C}^{d}$ & 1,5 \\
\hline HE 2347-4130 & $23: 50: 19.70-41: 14: 01.1$ & 15.16 & 4.07 & 44875 & 5.83 & 1.44 & 1.88 & $\mathrm{C}$ & \\
\hline HE 0414-5429 & $04: 15: 30.23-54: 21: 58.7$ & 14.60 & 3.86 & 44678 & 5.76 & $1.57^{*}$ & 1.96 & $\mathrm{C}^{d}$ & \\
\hline HE 0914-0314 & $09: 17: 15.62-03: 53: 57.3$ & 14.93 & 3.97 & 45136 & 5.79 & 1.65 & 1.94 & $\mathrm{C}^{d}$ & 1 \\
\hline BPS CS 22955-0024 & $20: 23: 50.26-25: 08: 28.8$ & $15.50^{c}$ & 4.04 & 44622 & 5.80 & 1.72 & 1.89 & $\mathrm{C} \& N$ & 4 \\
\hline HE $0155-3710$ & 01:58:01.44-36:56:21.9 & 15.08 & 4.24 & 41405 & 5.77 & 1.76 & 1.76 & $\mathrm{~N}$ & 3,7 \\
\hline HE 1136-1641 & $11: 38: 54.62-16: 58: 13.4$ & $14.82^{a}$ & 4.03 & 44646 & 5.80 & 1.81 & 1.88 & $\mathrm{C}$ & 2 \\
\hline HE $0958-1151$ & $10: 00: 42.64-12: 06: 00.0$ & $13.81^{a}$ & 3.29 & 44125 & $5.51^{*}$ & 1.85 & 2.18 & $\mathrm{C}^{d}$ & $1,2,4,5$ \\
\hline HE 0016-3212 & $00: 18: 53.22-31: 56: 01.7$ & 14.27 & 3.73 & 41674 & $5.70^{*}$ & 2.14 & 1.97 & $\mathrm{C} \& \mathrm{~N}$ & 3,6 \\
\hline HE 0031-5607 & 00:34:07.75 -55:51:05.9 & 15.45 & 5.37 & 41423 & 6.25 & $2.26^{*}$ & 1.31 & $\mathrm{~N}$ & 8 \\
\hline HE 1316-1834 & $13: 19: 16.93-18: 49: 52.0$ & 16.24 & 3.55 & 42811 & 5.56 & 2.32 & 2.05 & $\mathrm{~N}$ & 2,8 \\
\hline PG 2258+155 & $23: 00: 57.75+15: 48: 39.8$ & $15.14^{c}$ & 4.77 & 42084 & $6.08^{*}$ & $2.84^{*}$ & 1.56 & $\mathrm{~N}$ & 1,4 \\
\hline PG $1632+222$ & $16: 34: 16.09+22: 11: 40.9$ & $15.13^{b}$ & 4.77 & 39384 & 6.16 & 2.84 & 1.31 & $\mathrm{C}$ & $1,4,8$ \\
\hline HE $0001-2443$ & $00: 04: 31.01-24: 26: 21.1$ & 13.66 & 4.62 & 40975 & 5.94 & 2.97 & 1.60 & $\mathrm{~N}$ & 3 \\
\hline HZ 1 & $04: 50: 13.52+17: 42: 06.2$ & $12.60^{a}$ & 3.95 & 41344 & 5.68 & 3.00 & 1.87 & $\mathrm{~N}$ & 3,4 \\
\hline
\end{tabular}

1: Also in PG survey (Green et al. 1986). 2: Also in EC survey (Stobie et al. 1987). 3: Also in MCT survey (Demers et al. 1987). 4: McCook \& Sion (1999), misclassified as white dwarf. 5: Christlieb et al. (2001). 6: Possibly cool companion indicated by $B-J, J-K$ colours. 7: Possibly cool companion indicated by $B-J$ colour. 8 : Only one spectrum available

${ }^{a}$ From subdwarf data base (Østensen 2006). ${ }^{b}$ From SDSS $g^{\prime}$ and $r^{\prime}$ using the calibration of Smith et al. (2002). ${ }^{c}$ Photographic magnitude (SIMBAD). ${ }^{d}$ Weak $\mathrm{N}$ lines also present.

classified as an sdO star in the PG survey, six of our sdO stars are classified as sdB in the PG catalog. Five of them turn out to be helium deficient (see Sect. 5). It is likely that the helium deficient sdO stars are subsumed in the sdB class of the PG catalog; hence, the fraction of sdO stars is underestimated by Green et al. (1986).

The high spectral resolution of the UVES spectra allowed us to identify and measure not only hydrogen and helium lines, but also the metal line spectrum. Lines from highly ionised carbon and nitrogen are found in normal O-type stars. For a refined spectral classification, we thus used the presence or absence of characteristic absorption lines of carbon and nitrogen to divide our sample into several subclasses ("CN-scheme"). For carbon we used the strongest lines of C III (4650 A, multiplet 1, $4070 \AA$, mult. 16, and $4186.9 \AA$ of mult. 18) and of C IV (5801.33 $\AA$ and 
$5811.98 \AA$, mult. 1 , and $4658.3 \AA$, mult. 8). For nitrogen the strongest lines of N III we used (4640 $\AA$, mult. 2 and $4858 \AA$, mult. 9, $4379.11 \AA$ A, mult. 18).

We adopted the following classification scheme:

- Stars with C III and/or C IV visible in their spectra but with no N III were classified as " $\mathrm{C}$ ".

- Stars with N III and C III and/or C IV in their spectra were classified as "C\&N".

- The absence of C III and C IV and the presence of N III leads to the classification "N".

- With no lines of C III, C IV, and N III visible a star was classified as " 0 ".

This is not an optimised, quantitative classification scheme, but was intended to demonstrate that even such a qualitative subdivision already shows a correlation with the atmospheric parameters of our stars, as we outline below. The resulting classifications are presented in Table 1 . We checked whether the presence or absence of $\mathrm{C}$ and/or $\mathrm{N}$ lines might be significantly influenced by the signal-to-noise ratio $(\mathrm{S} / \mathrm{N})$ and found that the $\mathrm{S} / \mathrm{N}$ distribution of our spectra does not differ for different $\mathrm{CN}$-classes at low and intermediate $\mathrm{S} / \mathrm{N}$ values, for which it could be crucial. Hence the $\mathrm{CN}$ classification scheme is not significantly affected by noise.

\subsection{Search for companions of sdO stars}

In Paper I we found a significant fraction of sdB stars with cool companions ( 24 out of $76 \mathrm{sdB}$ stars). Therefore we searched for spectroscopic and photometric evidence for such companions amongst our sdO stars.

Only for one subluminous O star, HE 1502-1019 (alias PG 1502-103; EC 15026-1019), spectroscopic signatures for a cool companion were found, like the the G-band and the Mgtriplet. Ferguson et al. (1984) classified the companion star as spectral type K0.5. The weakness of the helium lines implies a very low helium abundance of about $\mathrm{He} / \mathrm{H}=0.001$. We estimate the effective temperature from a comparison to synthetic spectra (see Sect. 3) to be near $45000 \mathrm{~K}$.

HE 0301-3039 turns out to be radial velocity variable and its spectrum is also composite; it indicates that HE 0301-3039 is a binary consisting of two sdO stars with spectra dominated by helium lines, the first such system found (Lisker et al. 2004).

HE 1200-1924 (alias EC 12001-1924; Feige 54) shows a helium-dominated absorption-line spectrum typical of an sdO star. However, Balmer emission lines are superimposed - strong and broad $\mathrm{H} \alpha$, strong but narrow $\mathrm{H} \beta$ and weak $\mathrm{H} \gamma$ and $\mathrm{H} \delta$ emission. These emissions do not originate in the photosphere of the sdO star but may stem from a companion star. No spectral absorptions typical of a cool companion can be found. The $B-J$ and $J-K$ colours, however, are unusual for an sdO star (see below).

For these two objects, too few spectra are at hand to disentangle the individual spectra, so the quantitative spectral analysis of these objects has to be postponed until they become available.

We also searched for photometric evidence for companions by complementing the $B$-band fluxes with IR measurements from the 2MASS (Skrutskie et al. 2006) and DENIS ${ }^{1}$ catalogs. Photoelectric $B$ magnitudes (accurate to better than \pm 0 m.05) were taken from the subdwarf data base (Østensen 2006). If the former were unavailable, $B$ magnitudes are from the Hamburg-ESO survey (Wisotzki et al. 2000, accurate to \pm 0.2 ).

\footnotetext{
${ }^{1}$ http://cdsweb.u-strasbg.fr/denis.html
}

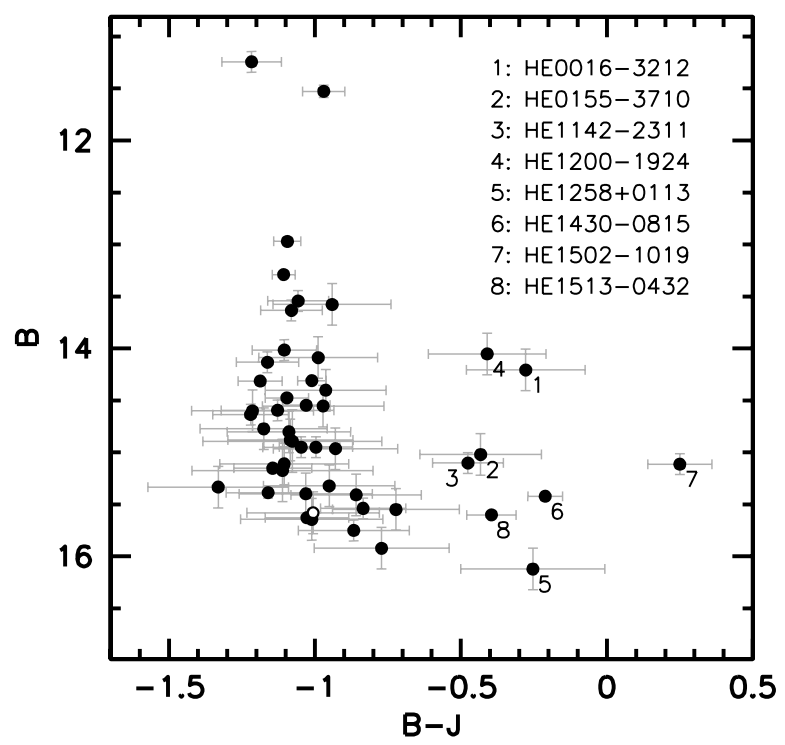

Fig. 1. Colour-magnitude diagram, showing $B$ magnitude versus optical-to-near-infrared colour $(B-J)$. Numbers denote objects that lie off the bulk of "normal" sdOs, implying a cool companion. The sdOsdO binary HE0301-3039 is shown as open circle. HE 1502-1019 and HE 1200-1924 are composite spectrum objects (see text).

The $J$ magnitudes and corresponding errors were provided by 2MASS for 51 stars and by DENIS for one star. Only the brightest stars were detected in the $K$ band. A colour-magnitude diagram is displayed in Fig. 1. Interstellar extinction and reddening were corrected for using the maps of Schlegel et al. (1998). As most of the stars are located at high galactic latitudes, $E(B-V)$ is small $\left(\leq 0^{\mathrm{m}} \cdot 12\right)$ except for the low-latitude star $\mathrm{HZ}$ 1. For the latter, the reddening as inferred from the Schlegel maps is overestimated, since the star lies near the Galactic plane $(z \approx 80 \mathrm{pc})$. Instead, we compared the measured $B-V$ to model predictions, yielding $E(B-V)=0.25$.

In Paper I we found that sdB stars with spectroscopic signatures of cool companions are redder than $B-J \approx-0.4$. Similarly, eight sdO stars in Fig. 1 lie at $B-J>-0^{\mathrm{m}} \cdot 5$ and clearly separate from the bulk of sdOs. We number those objects in the figure. Among them is HE 1200-1924, whose emission lines provide additional support for the existence of a cool companion to that $\mathrm{sdO}$ star.

For only 12 stars, $K$ band magnitudes are available to better than \pm 0.2 . Stark \& Wade (2003) studied the distributions of $J-K$ colours of sdB and sdO stars and find both to be doublepeaked, the sdO stars at $J-K=-0^{\mathrm{m}} \cdot 2$ and $+0^{\mathrm{m}} \cdot 2$, respectively, indicating that the latter sdO stars have cool companions. The $J-K$ measurements are available for five of the eight stars highlighted in Fig. 1. All five have positive $J-K$, indicating the presence of cool companions.

Judged from our experience with sdB stars, we can assume the blue spectra of these stars are not affected by light from the cool companion. Those stars are marked in Table 1, and the quantitative spectral analysis is performed in the same way as for the other stars (see Sect. 4).

\section{Stellar atmosphere modelling}

High-quality optical spectra like those from SPY require accurate theoretical counterparts as input for fitting routines. Only a few detailed quantitative spectral analyses have been carried out for the sdO class because of the complexity of their 
spectra. In addition deviations from local thermodynamic equilibrium (NLTE) have to be taken into account, because of their high effective temperatures, whereas the LTE assumption is reasonable for sdB stars. Therefore, only a few quantitative spectral analyses have been published (Dreizler et al. 1990; Thejll et al. 1994), with some conflicting results becoming apparent. The reliability of the NLTE calculation depends strongly on the quality and sophistication of the model atom and the atomic data used. The helium model atoms are of utmost importance for modelling sdO atmospheres. We used more detailed model atoms for He I and He II than in Dreizler et al. (1990) and constructed a new grid of atmospheric models and synthetic spectra using a stateof-the-art NLTE model atmosphere code.

An extensive grid of NLTE atmosphere models was calculated using the latest version of the PRO2 code (Werner \& Dreizler 1999) that employs a new temperature correction technique (Dreizler 2003). A new detailed model atom was constructed for helium to be appropriate for the sdO temperature regime, and 2700 partially line-blanketed NLTE model atmospheres consisting of hydrogen and helium were calculated, resulting in a grid of unprecedented coverage and resolution and extending from $30000 \mathrm{~K}$ to $100000 \mathrm{~K}$ in $T_{\text {eff }}$. The gravity ranges from $\log g=4.8$ to 6.4 and the helium abundance from $\log N_{\mathrm{He}} / N_{\mathrm{H}}=-4$ to +3 in order to match the diversity of the observed spectra. The step sizes are $2000 \mathrm{~K}$ from $30000 \mathrm{~K}$ to $52000 \mathrm{~K}$ and $5000 \mathrm{~K}$ from $55000 \mathrm{~K}$ to $100000 \mathrm{~K}$; 0.2 and $\sim 0.5$ dex, respectively.

As a test we carried out the spectral analyses of several programme stars using both the old grid of Dreizler et al. (1990) and the new one. The synthetic spectra from the new models match the observed profiles much better in all cases.

The differences of the current model grid compared to the one used by Dreizler et al. (1990) can be traced back to two major improvements. The resonance lines of He II could only be treated in detailed balance in the previous model grid. This eliminates the transition from the statistical equilibrium equations resulting in a stabler numerical behaviour. With an improved numerical stability of the code, this approximation is no longer necessary. Since the resonance lines of He II have a strong influence on the structure of the atmosphere of sdO stars, this improvement is the dominant effect. The second improvement since Dreizler et al. (1990) is the treatment of the level dissociation according to Hummer \& Mihalas (1988). This mainly affects higher line series members and is therefore important for a precise gravity determination.

\section{Spectral analysis: line profile fitting and error determination}

Atmospheric parameters $\left(T_{\text {eff }}, \log (g), \log (y), y=\mathrm{He} / \mathrm{H}\right.$ by number) were determined simultaneously by fitting the synthetic spectra to observed hydrogen and helium line spectra using an $\chi^{2}$-procedure (Napiwotzki et al. 1999).

For each line, the continuum level is determined and normalised to 1 , in order to compare it to the synthetic spectrum. For sdO stars with strong hydrogen Balmer lines, the $\mathrm{H} \alpha$ was excluded in the parameter determination because peculiarities in the $\mathrm{H} \alpha$ line core were often reported, perhaps caused by stellar winds (Heber et al. 2003b). Nevertheless $\mathrm{H} \alpha$ was kept in the final plot for visually examining any significant deviations from the model profiles. Those sdO stars showing no evidence of hydrogen from the visual inspection of the He II Pickering decrement required a modified fitting strategy. If hydrogen were present as a trace element, it could be detected best as a contribution of $\mathrm{H} \alpha$ to the He II Pickering line $6560 \AA$, but may be too weak to be visible to the eye. In a first step $T_{\text {eff }}$ and $\log (g)$ are derived by a fit using all lines including $6560 \AA$. Then a second fit is carried out to derive the hydrogen abundance keeping $T_{\text {eff }}$ and $\log (g)$ fixed from the first run. In a third step, new values for $T_{\text {eff }}$ and $\log (g)$ are derived while keeping the hydrogen abundance fixed. If the new values differ by more than $0.5 \%$, we repeat the procedure until convergence is achieved.

Two stars in our sample (DeHt 2 and K2-2) have already been analysed by Napiwotzki (1999) with similar NLTE model atmosphere techniques as presented here, and they are known to be central stars of planetary nebulae (CSPN). DeHt 2 was classified as a high-luminosity CSPN and $T_{\text {eff }}=117000 \mathrm{~K}$, $\log (g)=5.64$, and $\log (y)=-0.79$ were derived, whereas $\mathrm{K} 2-2$ was classified as a peculiar CSPN and $T_{\text {eff }}=67000 \mathrm{~K}$, $\log (g)=6.09$, and $\log (y)=-1.55$ resulted. These stars will not be discussed further in this paper.

For 46 out of the remaining 53 stars, the fit procedure converged to convincing line profile fits (as displayed in Fig. 2 for two typical cases). As can be seen, the agreement between the best-fit synthetic spectra and the observed ones is excellent.

However, for seven stars the fit procedure failed for various reasons. We discuss those stars in some detail below.

HE 1349-2320 (alias EC 13494-2320) and HE 1355-0622 (PG 1355-064): weak He I lines and strong He II lines indicate high temperatures. However, Balmer and He II lines cannot be matched simultaneously. The $\mathrm{H} \alpha$ lines display weak emission cores not reproduced by our models.

HE 1512-0331 (see Christlieb et al. 2001) displays very broad Balmer and He II lines with emission cores of He II, $6560 \AA$ and $4686 \AA$. Its gravity is too high for our model grid. Extrapolation suggests that its temperature may be near $80000 \mathrm{~K}$ and $\log (g)$ slightly below 7 . We regard the star as a DAO white dwarf.

HE 1518-0948 (alias PG 1518-098, see Moehler et al. 1990): Weak He I lines indicate high temperature, while Balmer and He II lines are narrow but strong indicating that it is a hot, helium-rich sdO star with low gravity. In fact, the attempts to fit its spectrum showed that the gravity must be lower than the lower limit of the grid, i.e. $\log (g)=4.8$ at a $T_{\text {eff }}$ near $60000 \mathrm{~K}$.

HE 2305-1155 displays strong and broad He I lines indicating a low $T_{\text {eff. Trials to match its spectrum indicated a very high }}$ gravity beyond the limit of our model grid (i.e. $\log (g)>6.4$ ).

HE 1008-179 (see Christlieb et al. 2001) is a very hot, high-gravity sdO star showing an $\mathrm{H} \alpha$ emission core. Individual Balmer lines can't be matched simultaneously (the so-called Balmer line problem, see Napiwotzki 1999). Attempts to fit its spectrum indicated that its parameters are probably beyond the high-temperature, high-gravity limit of the model grid, i.e. exceeding $T_{\text {eff }}=100000 \mathrm{~K}$ and $\log (g)=6.4$, and the star should be classified as an extremely hot white dwarf.

EC 11481-2303 has already been been analysed by Stys et al. (2000) who derived $T_{\text {eff }}=41790 \mathrm{~K}, \log (g)=5.84$, $\log (y)=-1.85$. The authors were unable to match the UV spectral energy distribution. The Balmer line problem is obvious in our optical spectra from the fit, which indicated a higher temperature $(\approx 50000 \mathrm{~K})$ and lower helium content $(\approx-2.8)$, but similar gravity than found by Stys et al. (2000). The Balmer line problem in very hot white dwarfs was traced back to metal line blanketing by Werner (1996). Hammer et al. (2003) analysed the UV spectrum of EC 11481-2303 and derived very large iron and nickel abundances of 10 to 100 times solar, causing a strong lineblanketing effect. Hence, the neglect of metal line-blanketing is 

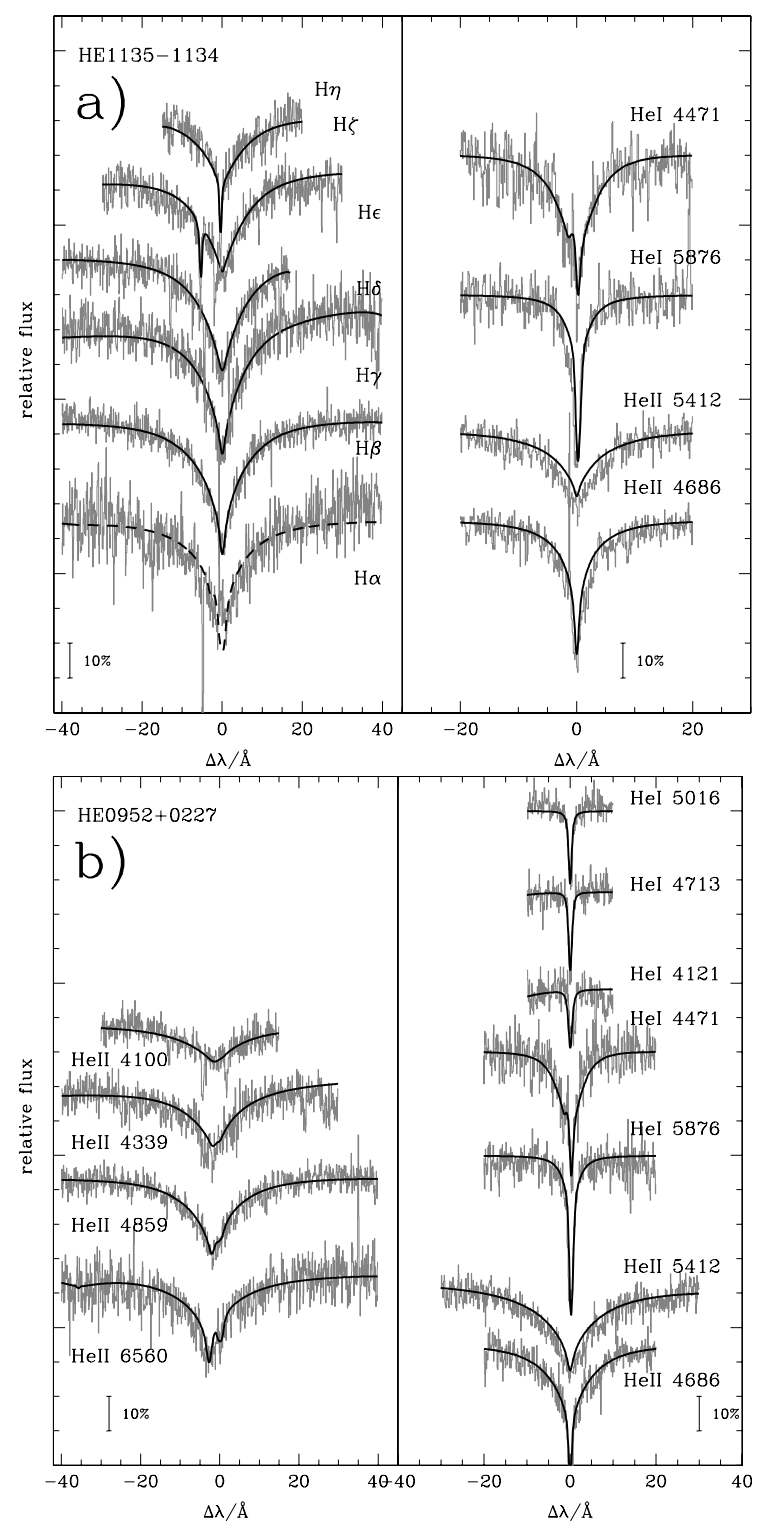

Fig. 2. Sample fit of two sdO star spectra (grey) using the new grid of synthetic NLTE spectra as described in Sect. 3. In HE 11351134 (top panel) hydrogen is more abundant than helium, whereas in HE 0952+0227 (bottom panel) hydrogen is a trace element only. The model spectra yielding the best fits are shown as black lines. For details see text.

the likely cause of our failure to match the optical spectra of this star.

Because of the peculiarities described and problems encountered in the fit procedure, we do not include these stars in the further discussion and are left with a working sample of 46 stars.

Since the SPY project was originally intended to search for radial velocity variable stars, two or more exposures of each star are mostly available. Thus the final values were calculated as the average of the individual fit results, weighted with the $S / N$ of the corresponding spectra. The final values are presented in Table 1. B magnitudes are from the Hamburg ESO objective prism survey (photographic, accurate to $\pm 0.2 \mathrm{mag}$, see Wisotzki et al. 2000) if not specified otherwise. We note that for seven stars of the working sample only a single (useful) exposure was available and denote them by " $S$ " in Table 1. Effective temperatures range from $36000 \mathrm{~K}$ to $78000 \mathrm{~K}$, gravities from $\log (g)=4.9$ to 6.4 , and
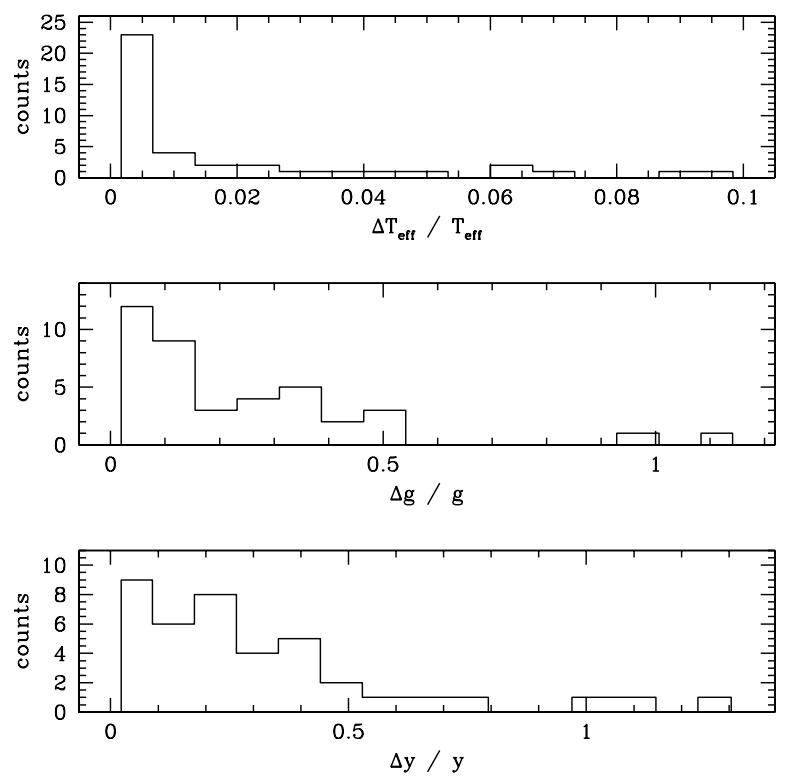

Fig. 3. Distribution of relative errors in atmospheric parameters, as derived from two or more exposures. From top to bottom the distributions for $T_{\mathrm{eff}}, g$, and $y=N_{\mathrm{He}} / N_{\mathrm{H}}$ are shown.

helium-to-hydrogen ratios from 0.0003 to more than 1000 . By adopting a canonical mass of $0.47 M_{\odot}$ for our programme stars, we can further derive the luminosity in solar units (Table 1).

The statistical $1-\sigma$-errors from the fit procedure are typically lower than $100 \mathrm{~K}, 0.04 \mathrm{dex}$, and $0.04 \mathrm{dex}$ for $T_{\text {eff }}, \log (g)$, and $\log (y)$, respectively, as a consequence of the high-resolution and the low noise level of our data. Nevertheless we decided to derive a more reliable estimate of the true errors on the basis of the existing two or more exposures for each star, where available. We determined the relative error for $T_{\text {eff }}, g$, and $y$ by taking the difference between the fit results of each exposure and dividing by the weighted mean of these fit results. Figure 3 shows the histogram distribution of the resulting relative errors. We find the $1-\sigma$-value (sample standard deviation) of these distributions to yield global relative errors of $\Delta T_{\text {eff }} / T_{\text {eff }}=0.026, \Delta g / g=0.25$, and $\Delta y / y=0.30$. This corresponds to $\Delta \log T_{\text {eff }}=0.011$, $\Delta \log (g)=0.097$ and $\Delta \log (y)=0.11$. We note that some relative errors are particularly large, i.e. more than 2- $\sigma$ (sample standard deviation) of these distributions. These so-called "outliers" are flagged in Table 1.

\section{Spectral analysis: results, trends, and biases}

As outlined in Edelmann et al. (2003) and Paper I, we shall search for trends in parameter space. We discuss the distributions of gravity, temperature, and helium abundance, taking the CN-classification scheme into account (see Sect. 2.1). As systematic differences in atmospheric parameters lead to selection biases, we discuss this issue at the end of this section.

\subsection{Atmospheric parameters and $C \& N$ line strengths}

The following conclusions can be drawn from the distribution of stars in the $T_{\text {eff }}-\log (y)$-diagram (Fig. 4):

(i) $\mathrm{SdO}$ stars with a helium abundance below solar, ranging from $0.0003 \leq \mathrm{He} / \mathrm{H} \leq 0.08$, are scattered in a wide range of effective temperatures from $35 \mathrm{kK} \lesssim T_{\text {eff }} \lesssim 80 \mathrm{kK}$, and no correlation can be found. 


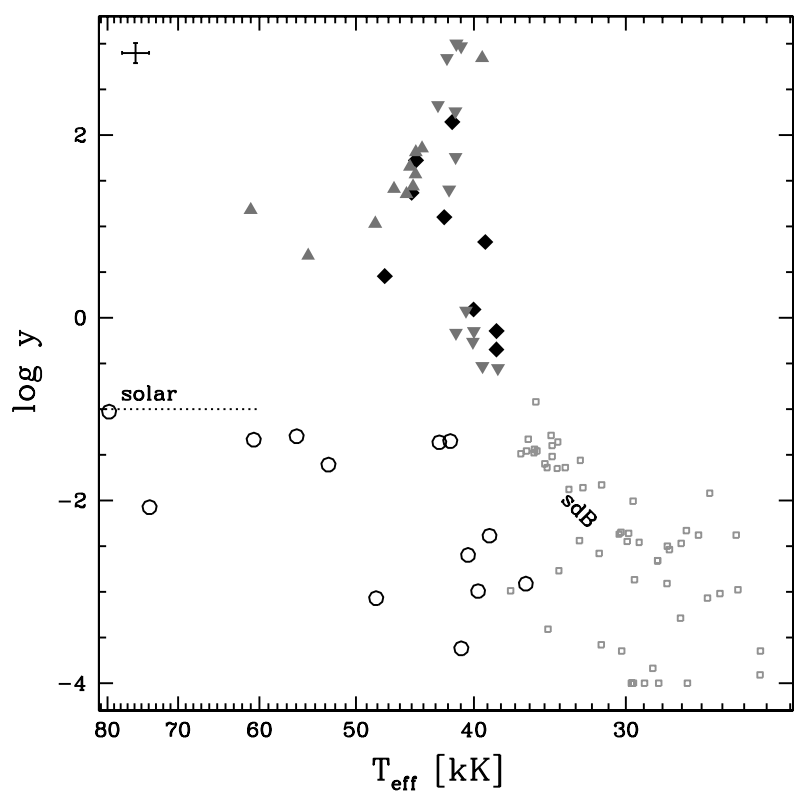

Fig. 4. Helium abundance versus effective temperature for $\operatorname{sdO}$ as shown by their $\mathrm{CN}$-classification and comparison to the sdB stars (open squares) from the SPY project (Lisker et al. 2005). Open circles denote stars of $\mathrm{CN}$ class 0 , filled triangles are either class $\mathrm{N}$ (upside down triangle) or class $\mathrm{C}$, whereas stars of class $\mathrm{C} \& \mathrm{~N}$ are marked by a filled diamond. The sdB stars are marked by open squares. Measurement uncertainties are given in the upper left corner.

(ii) $\mathrm{SdO}$ stars with a helium abundance exceeding the solar value, ranging from $0.1 \lesssim \mathrm{He} / \mathrm{H} \lesssim 1$, tightly cluster around $T_{\text {eff }} \approx 40 \mathrm{kK}$ and $\mathrm{He} / \mathrm{H} \approx 0.5$.

(iii) Above a helium abundance of $\mathrm{He} / \mathrm{H}=10$, the sdO stars show a clear dependence of the helium abundance on temperature: the helium abundance decreases with increasing $T_{\text {eff }}$, opposite to the general trend for $\mathrm{sdB}$ stars.

Most strikingly a clear correlation between helium abundance and $\mathrm{CN}$ class becomes apparent from Fig. 4. None of the sdO stars with subsolar helium content shows carbon and/or nitrogen lines and therefore all of them are of $\mathrm{CN}$ class 0 . The opposite is true for sdO stars with supersolar helium content - all of them show carbon and/or nitrogen lines and therefore are either of class $\mathrm{C}, \mathrm{N}$, or $\mathrm{C} \& \mathrm{~N}$.

This suggests that the sdO stars should be grouped into two classes according to helium content. Those with supersolar helium abundances will later be referred to as helium-enriched sdO stars, while those with subsolar helium abundances will be termed helium-deficient sdO stars.

Figures 5 and 6 show the distribution of the full SPY sample of sdO stars in the $T_{\text {eff }}-\log (g)$-plane, in comparison with the sdB stars from Paper I. The location of the EHB band (Dorman et al. 1993) is shown, along with the helium zero-age main sequence (He-ZAMS), which indicates the boundary beyond which no stable helium-core burning can be established (Paczyński 1971).

Both helium-deficient (Fig. 5) and helium-enriched (Fig. 6) sdO stars are found at higher temperatures than the sdB stars, most of them between $37 \mathrm{kK}$ and $47 \mathrm{kK}$. They clearly lie outside the EHB band, which is defined as the region between the zero-age EHB (ZAEHB) and the terminal age EHB (TAEHB) given by the evolutionary calculations of Dorman et al. (1993). A significant fraction (7 out of 33 ) of helium-enriched sdOs appear to lie below the helium main sequence. In view of the

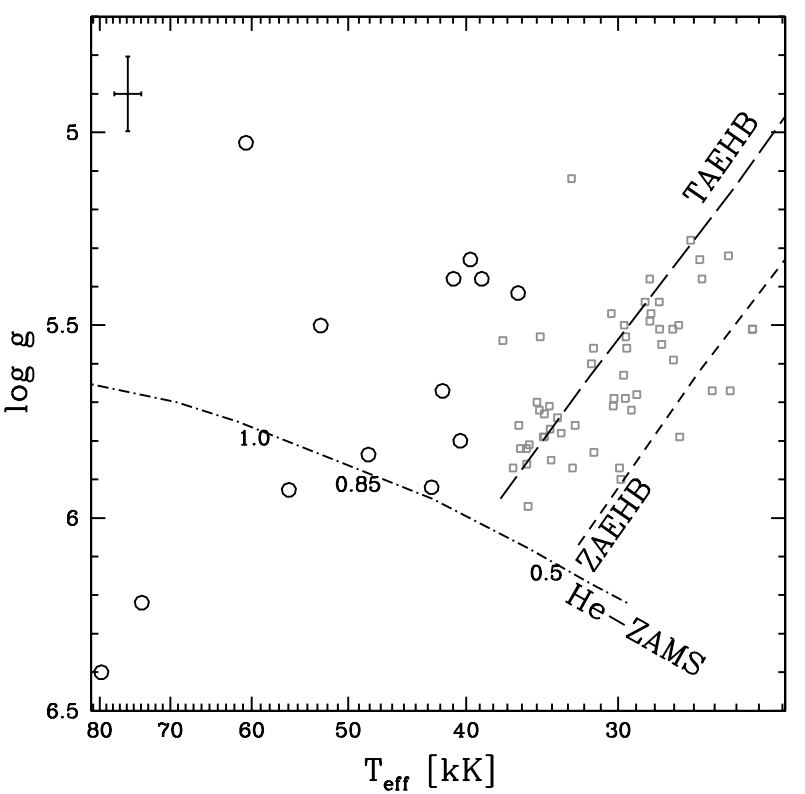

Fig. 5. Helium-deficient sdO stars: distribution of effective temperature and surface gravity. The location of the EHB band and the helium zeroage main sequence (He-ZAMS, labelled by stellar mass in solar units) are also indicated. The notations are the same as in Fig. 4. Measurement uncertainties are shown in the upper left corner.

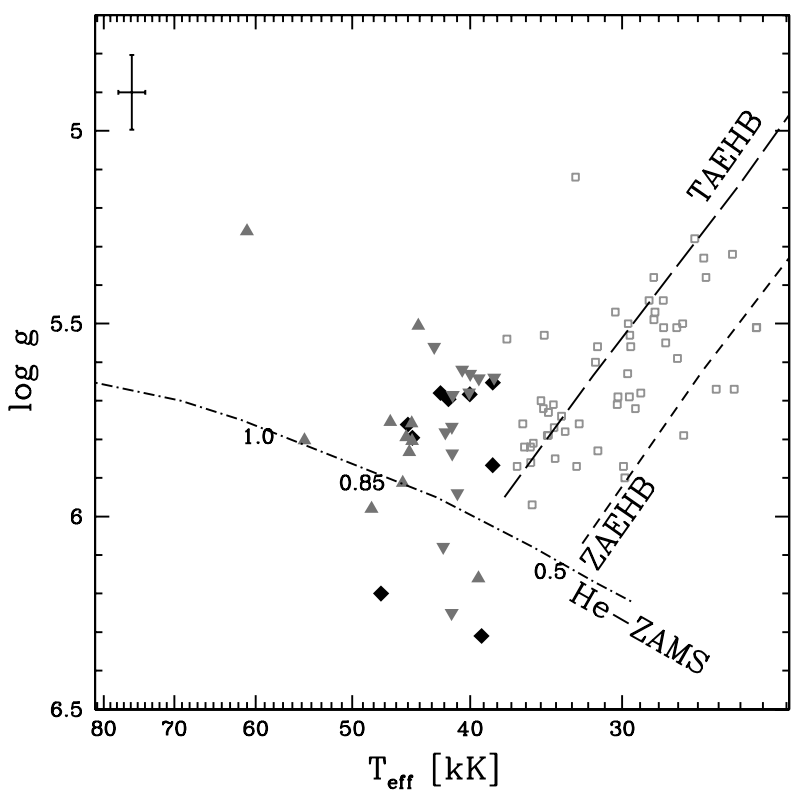

Fig. 6. Helium-enriched sdO stars: distribution of effective temperature and surface gravity. The location of the EHB band and the helium zeroage main sequence (He-ZAMS, labelled by stellar mass in solar units) are also indicated. The notations are the same as in Fig. 4. Measurement uncertainties are shown in the upper left corner.

non-Gaussian distribution of errors (see Sect. 3), their gravities might have been overestimated. Indeed, two stars are amongst the "outliers" and the results of another three are based on single spectra. It may therefore be premature to draw reliable conclusions. Additional observations are needed to derive more precise gravities.

While the helium-deficient $\mathrm{sdO}$ stars are scattered in a wide $T_{\text {eff }}-\log (g)$-range, most helium-enriched sdOs populate a relatively narrow region $\left(T_{\text {eff }}\right.$ from $\sim 40$ to $\sim 46 \mathrm{kK}$ and $\log (g)$ from $\sim 5.5$ to $\sim 5.9$ ). 


\subsection{Selection bias}

The target objects of SPY were selected from a magnitudelimited list of candidate white dwarfs. Therefore all subdwarfs present in the SPY dataset have only been included "by accident", because they were classified erroneously as white dwarfs mostly from low-resolution objective prism spectra. For the sdB stars, we found in Paper I that any selection effects cannot be significantly different from typical selection effects present in earlier studies of sdB stars. Besides the usual biases of magnitudelimited surveys, there may be another selection effect introduced by the SPY observing strategy. As the SPY project aimed at including a significant fraction of helium-rich white dwarfs, preference was given to DB candidates, many of which turned out to be sdO stars. There may thus be a bias in favour of selecting sdO stars compared to $s d B$ stars in the SPY project. This must be taken into account when the combined sdB and sdO sample from SPY is compared to predictions from evolutionary calculations.

\section{Evolutionary status}

As outlined above, the distribution of the programme stars in the $T_{\text {eff }}-\log (g)$-diagram (see Figs. 5 and 6), as well as with respect to $\mathrm{CN}$ class, suggests that helium-enriched sdO stars form a different population from the helium-deficient sdO stars. Published evolutionary scenarios try to explain the origin of $\mathrm{sdB}$ and $\mathrm{sdO}$ stars either by canonical or non-canonical evolution of single stars or by close binary evolution with mass exchange and common envelope episodes. We now test these scenarios by comparing our observational results to their predictions.

\subsection{Canonical evolution of single stars}

Canonical EHB models (e.g. Dorman et al. 1993) are characterised by a core mass of nearly half a solar mass and a tiny inert hydrogen-rich envelope. The core mass is fixed by the onset of the core helium flash at the tip of the RGB and depends only slightly on metalicity and helium abundance, so that the canonical core mass is restricted to a very narrow range of 0.46 to $0.5 M_{\odot}$. This configuration prevents an EHB star from ascending the AGB. The post-EHB evolution proceeds towards higher temperatures until the white-dwarf cooling track is reached and gravity increases.

The problem for the formation of EHB stars in this case is how almost the entire envelope of the RGB progenitor is lost at precisely the same time as the core reaches the mass required for the helium flash. Enhanced mass loss during or after the RGB has been postulated (D'Cruz et al. 1996), but no physical mechanism has been identified yet.

In Fig. 7 we compare the distribution of sdO stars to the position of the EHB-band and to post-EHB evolutionary tracks and find that none of the programme stars lies on the EHB-band. However, it is premature to conclude that they have evolved from the EHB. Most of the helium-enriched sdO stars cluster in a narrow region of the $T_{\text {eff }}-\log (g)$-diagram. The calculations of Dorman et al. (1993) indicate that the pace of evolution does not change very much through post-EHB evolution, so post-EHB evolution can't explain the clustering of helium-enriched $\mathrm{sdO}$ stars. Moreover, the post-EHB scenario fails to explain the surface enrichment of helium, and it also can't explain why the $\mathrm{C}$ and/or $\mathrm{N}$ lines in helium-enriched sdO stars are stronger than helium-deficient ones, since no dredge-up process is predicted.

The helium-deficient sdO stars, however, can be explained well by the post-EHB hypothesis as they have similarly low

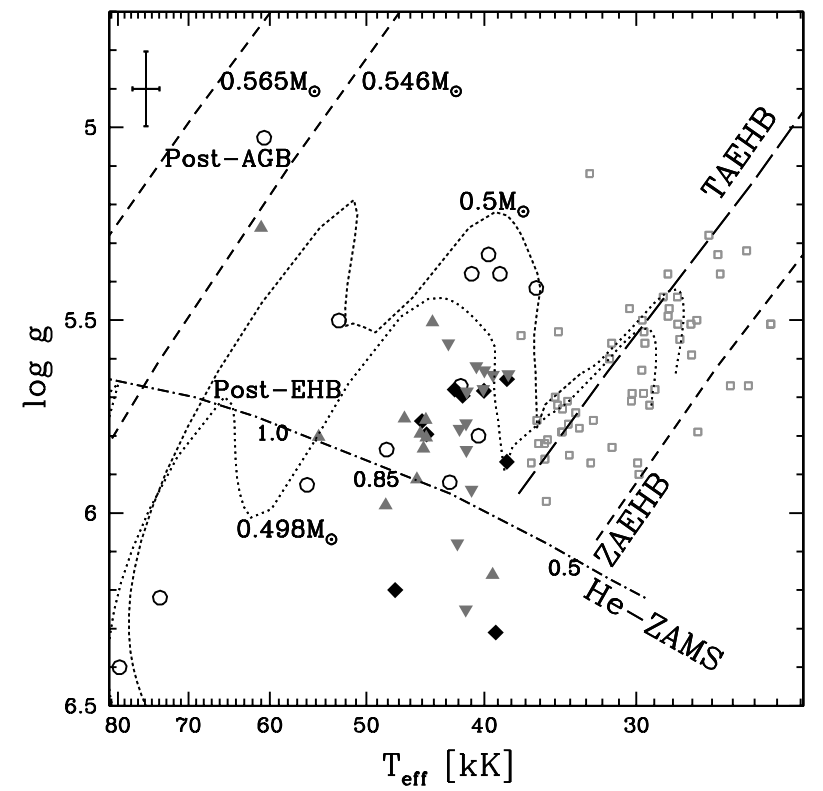

Fig. 7. Tracks for post-EHB evolution (black dotted lines) from Dorman et al. (1993) are superposed onto the $T_{\text {eff }}-\log (g)$-diagram for heliumdeficient and helium-enriched $\mathrm{sdO}$ stars, with core masses as indicated next to the tracks. The notations are the same as in Figs. 5 and 6. We also show post-AGB tracks (dashed lines) from Schönberner (1979, 1983) with white-dwarf (final stage) masses as indicated next to the tracks. The helium main sequence is labelled by stellar mass in solar units.

helium abundances as sdB stars. In addition they are scattered in the $T_{\text {eff }}-\log (g)$-diagram, so no slow-down in their evolution has to be invoked. Therefore it is reasonable that helium-deficient sdO stars are post-EHB stars that have evolved from sdB stars, while helium-enriched sdO stars have not.

Some of the more luminous sdO stars may not be related to the EHB at all, but may have evolved off the AGB. Therefore we included post-AGB evolutionary tracks from Schönberner $(1979,1983)$ in Fig. 7. The position of the helium-deficient sdO HE $1047-0637\left(T_{\text {eff }} \approx 60000 \mathrm{~K}, \log (g) \approx 5.0\right)$ is matched by these tracks, indicating a possible post-AGB evolutionary stage, while the helium-enriched sdO HE 1430-0815 ( $T_{\text {eff }} \approx$ $61000 \mathrm{~K}, \log (g) \approx 5.3$ ) may be either a post-AGB or a postEHB star. The short evolutionary timescales (about 30000 years from the AGB towards a pre-white dwarf, Schönberner 1979, 1983) drastically reduce the probability of finding true postAGB stars.

\subsection{Non-canonical evolution of single stars}

Since canonical single-star evolution can't explain the heliumenriched sdO stars, in particular their clustering in the $T_{\text {eff }}-$ $\log (g)$-plane, the helium enrichment and the $\mathrm{C}$, and/or $\mathrm{N}$ line strengths, other scenarios have to be investigated, such as the late hot-flasher scenario, in which the core helium flash occurs when the star has already left the RGB and is approaching the white dwarf cooling sequence (delayed helium-core flash). During the flash, He and C is dredged-up to the surface (Sweigart 1997a).

Figure 8 compares an evolutionary track for the late hotflasher scenario with the distribution of our helium-enriched sdO stars. The star encountered a late flash on its way from the RGB towards the white-dwarf regime. This late hot flash forces the star to land on or near the helium main sequence, i.e. at the extreme end of the EHB. The final composition of the envelope is 


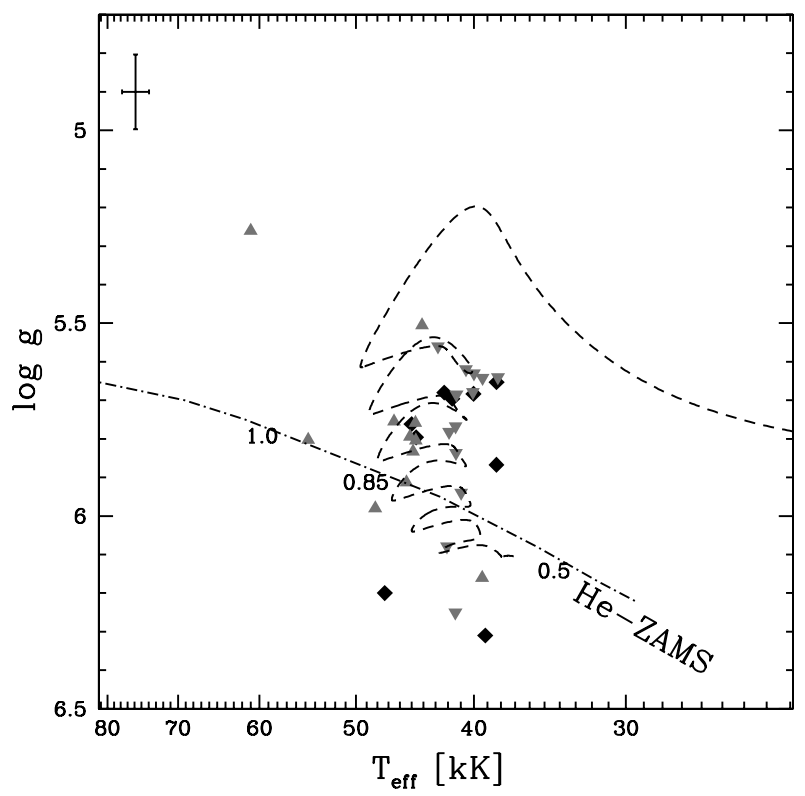

Fig. 8. Helium-enriched sdO stars: comparison with an evolutionary track for an EHB star formed by a delayed helium flash in the effective temperature vs. surface gravity plane. The track settles onto the helium main sequence (labelled by stellar mass in solar units). The symbols are the same as in Fig. 6.

helium-dominated with $Y=0.814$, complemented by hydrogen $(X=0.154)$ and enriched with carbon at $Z=0.032$ (or nitrogen if the hydrogen burning during the helium flash phase burns ${ }^{12} \mathrm{C}$ into ${ }^{14} \mathrm{~N}$; Sweigart 1997a). Indeed, most of our observed heliumenriched sdO stars lie near the model track, suggesting that they indeed may originate from this scenario (see also Lemke et al. 1997). However, the evolutionary time scales $\left(1.95 \times 10^{6} \mathrm{yr}\right.$ for the evolution shown in Fig. 8; Sweigart 1997a) are much shorter than for the core helium-burning phase. Accordingly the stars should accumulate near the end of the track, i.e. near the helium main sequence, which is not the case for our programme stars.

Although the late hot-flasher scenario can explain the helium enrichment and the line strengths of $\mathrm{C}$ and/or $\mathrm{N}$ lines as due to dredge up, it fails to reproduce the distribution of the stars in the $T_{\text {eff }}-\log (g)$-diagram in detail.

\subsection{Binary evolution}

As the evolutionary scenarios for single stars discussed above partially fail to explain the observed properties of heliumenriched sdO stars, we now focus on binary evolution. Two flavours of close binary evolution have been envisaged to explain $\mathrm{sdB}$ and sdO stars. The formation of core helium-burning EHB stars through close binary evolution has been investigated extensively by Han et al. $(2002,2003)$ using the binary population synthesis approach.

However, it may not be taken for granted that hot subluminous stars are indeed core helium-burning. They may form from red giants that left the RGB before igniting helium in the core and evolve through the EHB region as helium stars towards the white-dwarf cooling sequence, see Heber (2003). Such progenitors of helium-core white dwarfs have indeed been discovered. Heber et al. (2003a) found that the sdB star HD 188112 has a mass of $0.23 M_{\odot}$, which is too small to sustain helium burning.

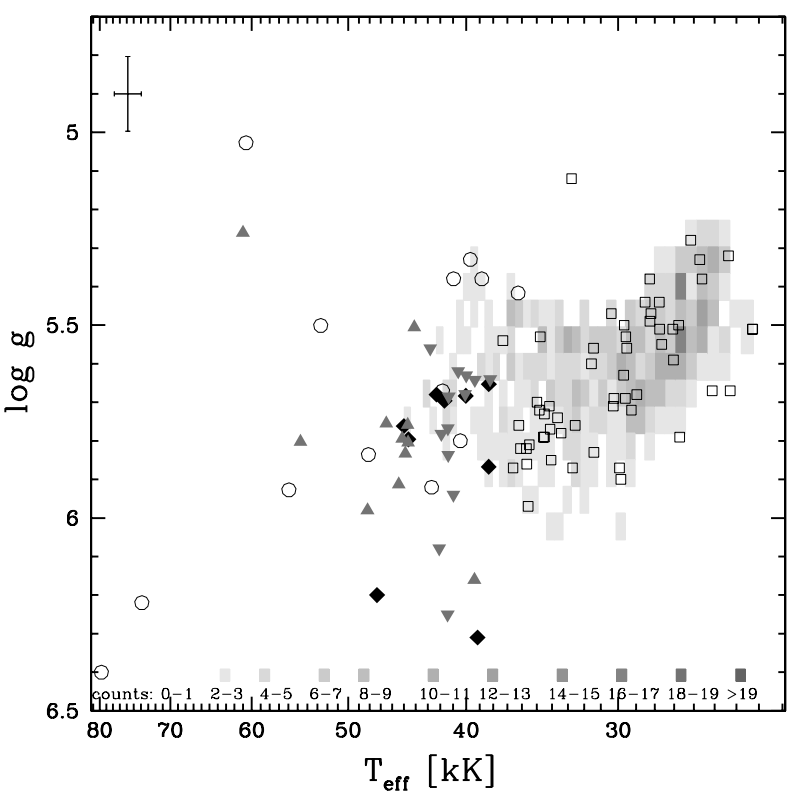

Fig. 9. Comparison of the atmospheric parameters of helium-deficient $\mathrm{sdO}$, helium-enriched $\mathrm{sdO}$, and $\mathrm{sdB}$ from the SPY project to simulation set No. 10 of (Han et al. 2003), which was found to match the $\mathrm{sdB}$ distribution of Paper I best. The theoretical predictions are shown as shaded $T_{\text {eff }}-\log (g)$-boxes, where a higher subdwarf density per box corresponds to darker shading. The grey scale is shown below the figures. The notations are the same as in Fig. 4.

\subsubsection{Binary evolution scenarios and the role of white-dwarf mergers}

In their binary population synthesis study Han et al. (2003, hereafter HPMM) showed that three channels are relevant to the formation of hot subluminous stars involving either commonenvelope ejection, stable Roche-lobe overflow, or a merger of two helium white dwarfs. Stable Roche-lobe overflow is predicted to lead to composite spectrum systems consisting of a hot subdwarf and a main sequence star. There are eight such binaries ( $\mathrm{sdO}+$ main sequence star) present amongst our programme stars.

In Paper I, we compared the atmospheric parameters of the SPY sdB stars to the HPMM models by using two diagnostic tools, namely the $T_{\text {eff }}-\log (g)$-diagram and the cumulative luminosity function. Our analysis of sdO stars extends the subdwarf sample to higher temperatures, which may allow the link between $\mathrm{sdB}$ and sdO stars to be studied. One would thus expect a comparison of our full sdB/sdO sample with the HPMM models to yield more robust results than in Paper I. However, we must observe selection biases as discussed in Sect. 5.2.

We present this comparison for the $T_{\text {eff }}-\log (g)$-diagram in Fig. 9. The simulation set No. 10 of HPMM was chosen because it came closest to the SPY-sdB distribution (see Paper I). We refer the reader to Paper I for more details about the general specifications of the HPMM simulations.

From the direct comparison of our derived $T_{\text {eff }}-\log (g)$ values to the HPMM simulations, we see two effects. First, sdO stars significantly exceed even the hottest temperatures that result from any HPMM simulation set, with stars reaching up to $80 \mathrm{kK}$. Even for the sdO stars with lower temperatures, no set is able to reproduce their rather wide range in surface gravity. HPMM therefore covers only $\leq 38 \%$ of all sdO stars. Second, by restricting our analysis to stars that come close to the HPMM predictions, i.e. those that are apparently connected with the $\mathrm{sdB}$ 
sample, a strong disagreement of the observational data with the simulation set becomes obvious: the relative amount of hot (sdO) and cool ( $\mathrm{sdB}$ ) stars differs significantly. Apart from possible limitations of the HPMM models (as discussed below in Sect. 7), there may be an observational bias in favour of sdO stars, rendering a quantitative comparison with HPMM, as performed for the sdB stars in Paper I, very difficult or even impossible.

Let us now focus on the white-dwarf merger channel, which is supposed to result in stars with very low hydrogen envelope mass. Hence stars resulting from mergers of two helium-core white dwarfs are expected to be found near the hot end of the HPMM distribution. Mergers can also produce stars in a wider range of masses than the other channels predict. The merging process probably induces lots of mixing of nuclear-processed material to the stellar surface of the remnant, potentially leading to a helium- and nitrogen-rich surface composition. Depending on the efficiency of nuclear burning and mixing, carbon may also be enriched at the surface. As these predictions match the observed properties of the helium-enriched sdO stars at least qualitatively, we regard the merger of helium core white dwarfs as a viable scenario. There are two predictions we can test: (i) stars formed from a merger should not be radial-velocity variable (unless they stem from triple systems) and (ii) the helium enrichment is accompanied by enrichment of nitrogen and/or carbon. While our CN-classification scheme (Sect. 2.1) is based on the presence of $\mathrm{C}$ and/or $\mathrm{N}$-lines in the spectrum, the latter issue requires a quantitative abundance analysis. We shall address these tests in forthcoming papers.

\subsubsection{Non core helium burning stars in close binary systems}

The evolution of RGB stars, whose envelopes get almost completely stripped by Roche-lobe overflow in a close binary system before helium burning starts, has been investigated e.g. by Driebe et al. (1998). These tracks were calculated from a $1 M_{\odot}$ model sequence starting from the pre-main sequence stage up through the RGB. High mass-loss rates were then adopted, and the evolution of the resulting helium star was followed. The remnant finally evolves into a helium-core white dwarf.

In Fig. 10 we compare the programme stars to the predictions of the evolutionary models of Driebe et al. (1998) for different masses. While the position in the $T_{\text {eff }}-\log (g)$-diagram of any of our programme stars can be matched by a post-RGB track of appropriate mass, it is striking that most of the helium-enriched sdO stars agree reasonably well with the theoretical predictions for a rather narrow mass range, i.e. between $0.3 M_{\odot}$ and $0.33 M_{\odot}$. Hence the distribution of helium-enriched sdO stars could be a sequence of low mass stars evolving into helium white dwarfs. It is also surprising that the predicted masses are close to the minimum mass for the helium main sequence, which might be purely coincidental.

However, the models of Driebe et al. (1998) predict rather thick hydrogen layers. Therefore, this scenario has difficulty explaining the high helium abundances at the stellar surface as observed in helium-enriched sdO stars. During the post-RGB evolution, hydrogen-shell flashes occur that possibly lead to a dredge-up of helium and nitrogen (Driebe et al. 1999). This may explain nitrogen strong-lined objects. It is, however, not evident how carbon-strong objects could be formed. Moreover, the models of Driebe et al. (1999) indicate that the occurrence of hydrogen flashes is constrained to a lower-mass range of 0.21 to $0.3 M_{\odot}$.

If the hydrogen were removed from the envelope by some unknown process, the evolution would be sped up considerably,

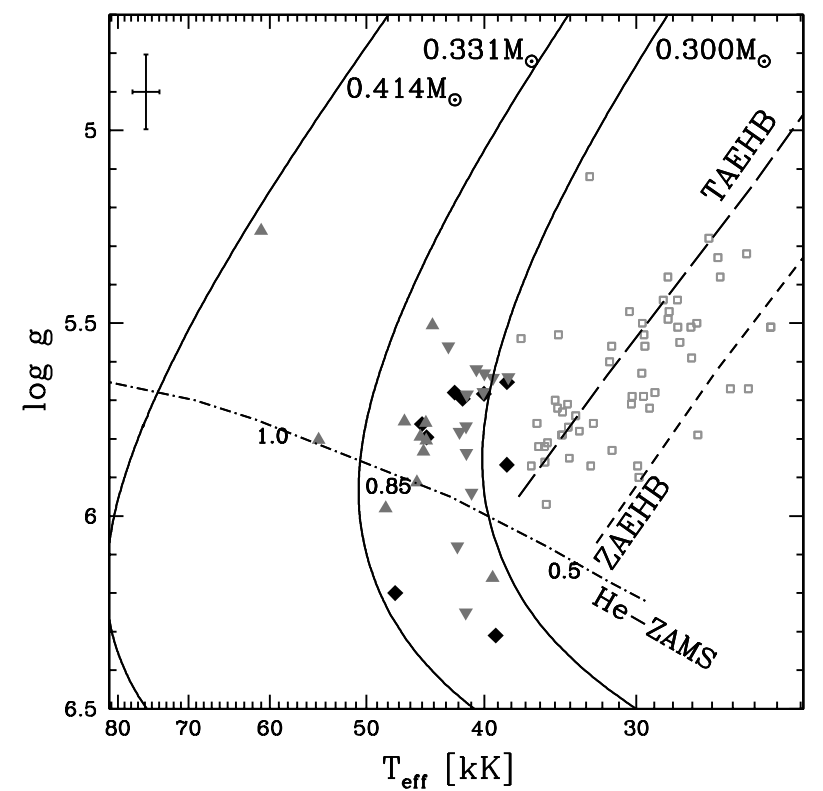

Fig. 10. Tracks for post-RGB evolution (solid lines) towards heliumcore white dwarfs of $0.3 M_{\odot}$ to $0.414 M_{\odot}$ (Driebe et al. 1998) are superposed onto the $T_{\text {eff }}-\log (g)$-diagram of helium-enriched sdO stars, with masses of the model star as indicated next to the tracks. The helium main sequence is labelled by stellar mass in solar units. The notations are the same as in Fig. 4.

because most of the luminosity is provided by the hydrogenburning shell, which will be extinguished if the hydrogen mass is too low. Rapid evolution reduces the detectability of such stars.

Therefore we regard the post-RGB scenario as unlikely. However, it is a viable scenario for explaining some of the helium-deficient sdO stars, since helium-core white-dwarf progenitors are known, albeit rare, amongst the sdB stars (e.g. HD 188112, Heber et al. 2003a).

\section{Summary and discussion}

We analysed the high-resolution optical spectra of 58 subluminous $\mathrm{O}$ stars and found spectroscopic and/or photometric evidence of cool companions to eight subluminous $\mathrm{O}$ stars. While HE 1502-1019 and HE 1513-0432 display only weak helium lines, the others have strong ones. HE 0301-3039 is a unique binary consisting of two sdO stars with spectra dominated by helium lines (Lisker et al. 2004). Evidence of cool companions to the other helium-enriched stars rests only on photometry. Nearinfrared spectroscopy is required for classifying them. As the photometric measurements for our sample are only available for 52 out of 58 stars, the fraction of sdO stars with cool companions may be slightly larger.

A grid of synthetic $\mathrm{H}-/ \mathrm{He}$-line spectra calculated from NLTE model atmospheres was used to derive the stars' atmospheric parameters. Reliable atmospheric parameters were obtained for 13 helium-deficient $\mathrm{sdO}$ and 33 helium-enriched sdO stars. A clearcut correlation between CNéclass and helium abundance was found. Helium-deficient sdO stars did not show any $\mathrm{C}$ and/or $\mathrm{N}$ lines, while all helium-enriched sdO stars do show either $\mathrm{C}$ or Nélines or both, suggesting that they form two different populations of stars. The helium-deficient sdO stars are scattered in the $T_{\text {eff }}-\log (g)$ diagram, whereas most helium-enriched sdO stars cluster in a narrow range.

Comparing the observed distribution to the predictions of evolutionary calculations for both single and close binary stars, 
we conclude that many helium-deficient sdO stars can be explained as evolved sdB stars. Both classes of star are heliumdeficient and have weak metal lines, which is caused by atmospheric diffusion processes.

Most of the helium-enriched $\mathrm{sdO}$ stars cluster in a narrow region of the $T_{\text {eff }}-\log (g)$-diagram at temperatures between $40 \mathrm{kK}$ and $50 \mathrm{kK}$. While diffusion is probably causing helium deficiency, it is unlikely to account for the helium enrichment. Non-standard evolutionary scenarios were therefore considered in addition. The predictions from both the late hotflasher scenario and the helium white-dwarf merger scenario are both roughly consistent with the observed distribution of heliumenriched $\mathrm{sdO}$ stars but do not match them in detail. The occurrence of both a delayed helium core flash, as well as the merger of two helium white dwarfs, may explain the helium enrichment. In these cases carbon and/or nitrogen can be dredged up to the stellar surface, which would explain the strength of the $\mathrm{C}$ and/or $\mathrm{N}$ lines in helium-enriched sdO stars.

Some high gravity helium-enriched $\mathrm{sdO}$ stars may lie below the helium main sequence, which is at variance with any core helium burning model. Therefore, we considered models for post-RGB stars with inert helium cores that evolve through the $\mathrm{sdB} / \mathrm{sdO}$ regime in the $T_{\text {eff }}-\log (g)$ diagram into helium-core white dwarfs. This is the only scenario that can explain stars lying below the helium main sequence. However, it is not obvious how the helium enrichment is brought about, since the hydrogen envelopes of post-RGB models have to be relatively thick or the stars would otherwise evolve too fast to be observable in large quantities. If the existence of a population of high gravity sdO stars below the helium main sequence could be confirmed by surveys with a larger sample size, a possible explanation could be provided by the post-RBG scenario.

Our conclusions can be tested by measuring the binary frequency and the $\mathrm{C}$ and $\mathrm{N}$ abundances. If helium-deficient $\mathrm{sdO}$ stars are evolved sdB stars, their binary fractions should be the same. As about $40 \%$ of the sdB stars are in close binaries with periods below 10 days (Napiwotzki et al. 2004), we expect 5 helium-deficient sdO stars in our sample to have a radial velocity that varies on a time scale of 10 days or less. An investigation of the binary frequency is underway in our group. If helium-enriched $\mathrm{sdO}$ stars result from mergers, no radial velocity variations would be expected (except for objects arising from triple stars). If material processed by nuclear burning is dredged up, the helium enrichment is expected to be accompanied by enrichment of nitrogen and/or carbon. While our CN-classification scheme (Sect. 2.1) is based on the presence of $\mathrm{C}$ and/or $\mathrm{N}$-lines in the spectrum, the latter issue requires a quantitative abundance analysis to test the late hot-flasher and the merger scenarios.

In addition, improvements to our analyses of the SPY-sample are required for a more detailed comparison with predictions of evolutionary calculations. The role of metal-line blanketing in the NLTE model atmospheres needs to be investigated. Due to the complexity of the problem, only a few studies of NLTE metal line blanketing in sdO star atmospheres are available (Haas et al. 1996; Lanz et al. 1997; Deetjen 2000), which indicates that the effective temperatures may have to be reduced. According to these investigations, the most important contribution to line blanketing stems from the iron group elements. However, their abundances can be derived only from ultraviolet spectra. The observed diversity of carbon and nitrogen line strengths indicates that metals have to be treated carefully on a star-by-star basis.

Apparently, the SPY sample suffers from observational selection biases. We found it likely that sdO stars were selected preferentially for the SPY target list, so that sdO stars may be overrepresented with respect to sdB stars. To this end the Sloan Digital Sky Survey (SDSS) is a promising source of hot subdwarf stars, as its selection criteria are very different from that of SPY. We have already begun a spectroscopic analysis of SDSS sdO stars (Heber et al. 2006), which will contribute significantly towards distinguishing between the various evolutionary scenarios and hypotheses outlined above.

Acknowledgements. T.L. gratefully acknowledges support by the Swiss National Science Foundation. R.N. is supported by a PPARC Advanced Fellowship. We thank Iris Traulsen and Thomas Rauch for their help in running the model atmosphere codes, Heiko Hirsch for double-checking the CNclassification, and Roy Østensen for creating the subdwarf data base (Østensen 2006), which we used extensively. This publication makes use of data products from the Two Micron All Sky Survey, which is a joint project of the University of Massachusetts and the Infrared Processing and Analysis Center/California Institute of Technology, funded by the National Aeronautics and Space Administration and the National Science Foundation (Skrutskie et al. 2006). This publication makes use of the VizieR database of astronomical catalogs (Ochsenbein et al. 2000).

\section{References}

Ahmad, A., \& Jeffery, C. S., 2003, A\&A, 402, 335

Ahmad, A., \& Jeffery, C. S. 2005, In 14th European Workshop on White Dwarfs, ed. D. Koester, S. Moehler, ASP Conf. Ser. 334, 291

Baschek, B., \& Norris, J., 1975, ApJ, 199, 694

Brown, T. M., Sweigart, A. V., Lanz, T., Landsman, W. B., \& Hubeny, I. 2001, ApJ, 562, 368

Charpinet, S., Brassard, P., Fontaine, G. 2004, Ap\&SS, 291, 395

Christlieb, N., Wisotzki, L., Reimers, D., et al. 2001, A\&A, 366, 898

D'Cruz, N. L., Dorman, B., Rood, R. T., \& O'Connell, R. W., 1996, ApJ, 466, 359

Deetjen, J. L., 2000, A\&A, 360, 281

Demers, S., Fontaine, G., Wesemael, F., Lamontagne, R., \& Irwin, M. J. 1987, in Second Conference on Faint Blue Stars, ed. A. G. D. Philip, D. S. Hayes, J. W. Liebert, IAU Coll. 95, 497

Dorman, B., Rood, R. T., \& O'Connell, R. W. 1993, ApJ, 419, 596

Dreizler S., 2003, in Stellar Atmosphere Modeling, ed. I. Hubeny, D. Mihalas, K. Werner, ASP Conf. Ser. 288, 69

Dreizler, S., Heber, U., Werner, K., Moehler, S., \& de Boer, K. S. 1990, A\&A, 235,234

Driebe, T., Schönberner, D., Blöcker, T., \& Herwig, F. 1998, VizieR Online Data Catalog, 333, 90123

Driebe, T., Blöcker, T., Schönberner, D., Herwig, F. 1999, A\&A, 350, 89

Drilling, J. S., Moehler, S., Jeffery, C. S., Heber, U., \& Napiwotzki, R. 2003, In The Garrison Festschrift, ed. R. O. Gray, C. J. Corbally, \& A. G. D. Philip, 27

Edelmann H., Heber, U., Hagen, H. J., et al. 2003, A\&A, 400, 939

Ferguson, D. H., Green, R. F., \& Liebert, J. 1984, ApJ, 287, 320

Geier, S., Heber, U., Przybilla, N., \& Kudritzki, R. P. 2006, Baltic Astron., 15, 243

Green, R. F., Schmidt, M., Liebert, J. 1986, ApJS, 61, 305

Greenstein, J. L., Sargent, A. I. 1974, ApJS, 28, 157

Groth, H. G., Kudritzki, R. P., Heber, U. 1985, A\&A, 152, 107

Haas, S., Dreizler, S., Heber, U., Jeffery, S., \& Werner, K. 1996, A\&A, 311, 669

Hammer, N. J., Deetjen, J. L., Dreizler, S., Werner, K., Kruk, J. W. 2003, in White Dwarfs, ed. D. de Martino, R. Silvotti, J. E. Solheim, \& R. Kalytis, NATO ASIB Proc., 105, 103

Han, Z., Podsiadlowski, P., Maxted, P. F. L., Marsh, T. R., \& Ivanova, N., 2002, MNRAS, 336, 449

Han, Z., Podsiadlowski, P., Maxted, P. F. L., \& Marsh, T. R., May 2003, MNRAS, 341,669

Heber, U., 1986, A\&A, 155, 33

Heber U., 2003, in White Dwarfs, ed. D. de Martino, R. Silvotti, J. E. Solheim, \& R. Kalytis, NATO ASIB Proc. 105, 49

Heber, U., \& Jeffery, C. S. 1992, LNP 401: The Atmospheres of Early-Type Stars

Heber, U., Edelmann, H., Lisker, T., \& Napiwotzki, R. 2003a, A\&A, 411, L477 Heber, U., Maxted, P. F. L., Marsh, T. R., Knigge, C., \& Drew, J. E., 2003b, in Stellar Atmosphere Modeling, ASP Conf. Ser. 288, 251

Heber, U., Hirsch, H., Ströer A., et al. 2006, Baltic Astron., 15, 91

Hummer, D. G., \& Mihalas, D. 1988, ApJ, 331, 794

Jeffery, C. S., Drilling, J. S., Harrison, P. M., Heber, U., \& Moehler, S. 1997, A\&AS, 125,501 
Koester, D., Napiwotzki, R., Christlieb, N., et al. 2001, A\&A, 378, 556

Lanz, T., Hubeny, I., \& Heap, S. R. 1997, ApJ, 485, 843

Lemke, M., Heber, U., Napiwotzki, R., Dreizler, S., \& Engels, D. 1997, in The Third Conference on Faint Blue Stars, 375

Lisker, T., 2003, Heiße unterleuchtkräftige Sterne aus dem SPY-Projekt, Master's thesis, University of Erlangen-Nürnberg

Lisker, T., Heber, U., Napiwotzki, R., et al. 2004, Ap\&SS, 291, 351

Lisker, T., Heber, U., \& Napiwotzki, R., et al. 2005, A\&A, 430, 223

Maxted, P. F. L., Marsh, T. R., \& North, R. C. 2000, MNRAS, 317, L41

Maxted, P. F. L., Heber, U., Marsh, T. R., \& North, R. C. 2001, MNRAS, 326 1391

McCook, G. P., \& Sion, E. M. 1999, ApJS, 121, 1

Moehler, S., Richtler, T., de Boer, K. S., Dettmar, R. J., \& Heber, U. 1990, A\&AS, 86, 53

Moehler, S., Sweigart, A. V., Landsman, W. B., Hammer, N. J., \& Dreizler, S. 2004, A\&A, 415, 313

Morales-Rueda, L., Maxted, P. F. L., Marsh, T. R., Kilkenny, D., \& O’Donoghue D. 2006, Baltic Astron., 15, 187

Napiwotzki, R. 1999, A\&A, 350, 101

Napiwotzki, R., Green, P. J., \& Saffer, R. A. May 1999, ApJ, 517, 399

Napiwotzki, R., Christlieb, N., Drechsel, H., et al. 2001, Astron. Nachr., 322, 411

Napiwotzki, R., Karl, C. A., Lisker, T., et al. 2004, Ap\&SS, 291, 321
Ochsenbein, F., Bauer, P., \& Marcout, J. 2000, A\&AS, 143, 23

Østensen, R. H. 2006, Baltic Astron., 15, 85

Paczyński, B. 1971, Acta Astron., 21, 1

Schlegel, D. J., Finkbeiner, D. P., Davis, M., 1998, ApJ, 500, 525

Schönberner, D., 1979, A\&A, 79, 108

Schönberner, D., 1983, ApJ, 272, 708

Skrutskie, M. F., Cutri, R. M., Stiening, R., et al., 2006, AJ, 131, 1163

Smith, J. A., Tucker, D. L., Kent, S., et al., 2002, AJ, 123, 2121

Stark, M. A., \& Wade, R. A., 2003, AJ, 126, 1455

Stobie, R. S., Morgan, D. H., Bhatia, R. K., Kilkenny, D., \& O’Donoghue, D. 1987, in Second Conference on Faint Blue Stars, ed. A. G. D. Philip, D. S. Hayes, \& J. W. Liebert, IAU Coll., 95, 493

Stys, D., Slevinsky, R., Sion, E. M., et al. 2000, PASP, 112, 354

Sweigart, A. V., 1997a, ApJ, 474, L23

Sweigart, A. V., 1997b, in The Third Conference on Faint Blue Stars ed. A. G. D. Philip, J. Liebert, R. Saffer, \& D. S. Hayes, 3

Thejll, P., Bauer, F., Saffer, R., et al. 1994, ApJ, 433, 819

Werner, K. 1996, ApJ, 457, L39

Werner, K., \& Dreizler, S. 1999, J. Comp. Appl. Math., 109, 65

Wesemael, F., Winget, D. E., Cabot, W., van Horn, \& H. M., \& Fontaine G. 1982, ApJ, 254, 221

Wisotzki, L., Koehler, T., Groote, D., \& Reimers, D. 1996, A\&AS, 115, 227

Wisotzki, L., Christlieb, N., Bade N., et al. 2000, A\&A, 358, 77 